EPJ manuscript No.

(will be inserted by the editor)

\title{
Non-concave fundamental diagrams and phase transitions in a stochastic traffic cellular automaton
}

\author{
Sven Maerivoet and Bart De Moor \\ Department of Electrical Engineering ESAT-SCD (SISTA) \\ Katholieke Universiteit Leuven \\ Kasteelpark Arenberg 10, 3001 Leuven, Belgium \\ Phone: +32 (0)16 321709 Fax: +32 (0)16 321970 \\ URL: http://www.esat.kuleuven.ac.be/scd \\ e-mail: sven.maerivoet@esat.kuleuven.ac.be
}

Received: June 11, 2004 / Revised version: July 10, 2018

\begin{abstract}
Within the class of stochastic cellular automata models of traffic flows, we look at the velocity dependent randomization variant (VDR-TCA) whose parameters take on a specific set of extreme values. These initial conditions lead us to the discovery of the emergence of four distinct phases. Studying the transitions between these phases, allows us to establish a rigorous classification based on their tempospatial behavioral characteristics. As a result from the system's complex dynamics, its flow-density relation exhibits a non-concave region in which forward propagating density waves are encountered. All four phases furthermore share the common property that moving vehicles can never increase their speed once the system has settled into an equilibrium.
\end{abstract}

PACS. 02.50.-r Probability theory, stochastic processes, and statistics - 05.70.Fh Phase transitions: general studies $-45.70 . \mathrm{Vn}$ Granular models of complex systems; traffic flow - 89.40.-a Transportation

\section{Introduction}

In the field of traffic flow modeling, microscopic traffic simulation has always been regarded as a time consuming, complex process involving detailed models that describe the behavior of individual vehicles. Approximately a decade ago, however, new microscopic models were being developed, based on the cellular automata programming paradigm from statistical physics.

The seminal work done by Nagel and Schreckenberg in the construction of their stochastic traffic cellular automaton (i.e., the STCA) 1, led to a global adoptation ' of the TCA modeling scheme. One of the artefacts associated with this STCA model, is that it gives rise to (many) unstable traffic jams [2]. A possible approach to achieve stable traffic jams, is to reduce the outflow from such jams, which can be accomplished by implementing so-called slow-to-start behavior. The name is derived from the fact that vehicles exiting jam fronts are obliged to wait a small amount of time. One implementation is based on making the noise parameter dependent on the velocity of a vehicle, leading to the development of the velocity dependent randomization (VDR) TCA. This TCA model moreover exhibits metastability and hysteresis phenomena 3 .

In this context, our paper addresses the VDR-TCA slow-to-start model whose parameters take on a specific set of extreme values. Even though this bears little di- rect relevance for the understanding of traffic flows, it will lead to an induced 'anomalous' behavior and complex system dynamics, resulting in four distinct emergent phases. We study these on the basis of the system's relation between density and flow (i.e., the fundamental diagram) which exhibits a non-concave region where, in contrast to the properties of congested traffic flow, density waves are propagated forwards. We finally investigate the system's tempo-spatial evolution in each of these four phases.

Although some related studies exist (e.g., 4, 5, 6, 7, 8 9) , the special behavior discussed in this paper has not been reported as such, as most previously done research is largely devoted to empirical and analytical discussions about these TCA models, operating under normal conditions. To strengthen our claims, we compare our results with the existing literature at the end of this paper.

In section 2 we briefly describe the concept of a traffic cellular automaton and the experimental setup used when performing the simulations. The selected VDR-TCA model is presented in section [3] as well as an account of some behavioral characteristics under normal operating conditions. The results of our various experiments with more complex system dynamics are subsequently presented and extensively discussed in section 4 after which the paper concludes with a comparison with existing literature in section [5 and a summary in section [6 


\section{Experimental setup}

In this section, we introduce the operational characteristics of a standard single-lane traffic cellular automaton model. To avoid confusion with some of the notations in existing literature, we explicitly state our definitions.

\subsection{Geometrical description}

Let us describe the operation of a single-lane traffic cellular automaton as depicted in Fig. 11 We assume $N$ vehicles are driving on a circular lattice containing $K$ cells, i.e., periodic boundary conditions (each cell can be occupied by at most one vehicle at a time). Time and space are discretized, with $\Delta T=1 \mathrm{~s}$ and $\Delta X=7.5 \mathrm{~m}$, leading to a velocity discretization of $\Delta V=27 \mathrm{~km} / \mathrm{h}$. Furthermore, the velocity $v_{i}$ of a vehicle $i$ is constrained to an integer in the range $\left\{0, \ldots, v_{\max }\right\}$, with $v_{\max }$ typically 5 cells $/ \mathrm{s}$ (corresponding to $135 \mathrm{~km} / \mathrm{h}$ ).

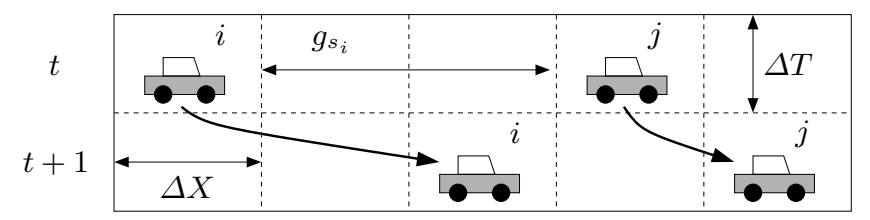

Fig. 1. Schematic diagram of the operation of a single-lane traffic cellular automaton (TCA); here, the time axis is oriented downwards, the space axis extends to the right. The TCA's configuration is shown for two consecutive time steps $t$ and $t+1$, during which two vehicles $i$ and $j$ propagate through the lattice. Without loss of generality, we denote the number of empty cells in front of vehicle $i$ as its space gap $g_{s_{i}}$.

Each vehicle $i$ has a space headway $h_{s_{i}}$ and a time headway $h_{t_{i}}$, defined as follows:

$$
\begin{aligned}
h_{s_{i}} & =L_{i}+g_{s_{i}}, \\
h_{t_{i}} & =\rho_{i}+g_{t_{i}} .
\end{aligned}
$$

In these definitions, $g_{s_{i}}$ and $g_{t_{i}}$ denote the space and time gaps respectively; $L_{i}$ is the length of a vehicle and $\rho_{i}$ is the occupancy time of the vehicle (i.e., the time it 'spends' in one cell). Note that in a traffic cellular automaton the space headway of a vehicle is always an integer number, representing a multiple of the spatial discretisation $\Delta X$ in real world measurement units. So in a jam, it is taken to be equal to the space the vehicle occupies, i.e., $h_{s_{i}}=1$ cell.

Local interactions between individual vehicles in a traffic stream are modeled by means of a rule set. In this paper, we assume that all vehicles have the same physical characteristics. The system's state is changed through synchronous position updates of all the vehicles, based on a rule set that reflects the car-following behavior.

Most rule sets of TCA models do not use the space headway $h_{s_{i}}$ or the space gap $g_{s_{i}}$, but are instead based on the number of empty cells $d_{i}$ in front of a vehicle $i$. Keeping equation (11) in mind, we therefore adopt the convention that, for a vehicle $i$ its length $L_{i}=1$ cell. This means that when the vehicle is residing in a compact jam, its space headway $h_{s_{i}}=1$ cell and its space gap is consequently $g_{s_{i}}=0$ cells. This abstraction gives us a rigorous justification to formulate the TCA's update rules more intuitively using space gaps.

\subsection{Performing measurements}

In order to characterize the behavior of a TCA model, we perform global measurements on the system's lattice. These measurements are expressed as macroscopic quantities, defining the global density $k$, the space mean speed $\bar{v}_{s}$, and the flow $q$ as:

$$
\begin{aligned}
k & =\frac{N}{K}, \\
\bar{v}_{s} & =\frac{1}{N} \sum_{i=1}^{N} v_{i}, \\
q & =k \bar{v}_{s} .
\end{aligned}
$$

The above measurements are calculated every time step, and they should be averaged over a large measurement period $T_{\text {sim }}$ in order to allow the system to settle into an equilibrium.

Correlation plots of these aggregate quantities lead to time-independent graphs conventionally called 'fundamental diagrams'. And although we should more correctly refer to our measurements as points in a certain phase space (e.g., the $(k, q)$ phase space), we will still use the terminology of 'fundamental diagram' in the remainder of this paper when we are in fact referring to this phase space.

The previous global macroscopic measurements (density, average speed, and flow) from equations (3), (4), and (5), can be related to the microscopic equations (11) and (2) as follows:

$$
\begin{aligned}
& k \propto \bar{h}_{s}^{-1}, \\
& q \propto \bar{h}_{t}^{-1},
\end{aligned}
$$

with $\bar{h}_{s}$ and $\bar{h}_{t}$ the average space and time headway respectively. Note that with respect to the time gaps and time headways, we will work in the remainder of this paper with the median instead of the arithmetic mean because the former gives more robust results when $h_{t_{i}}, g_{t_{i}} \rightarrow+\infty$ for a vehicle $i$.

All the fundamental diagrams in this paper, were calculated using systems of $10^{3}$ cells. The first $10^{3} \mathrm{~s}$ of each simulation were discarded in order to let initial transients die out; the system was then updated for $T_{\text {sim }}=10^{4} \mathrm{~s}$. 
For a deeper insight into the behavior of the space mean speed $\bar{v}_{s}$, the average space gap $\bar{g}_{s}$, and the median time gap $\bar{g}_{t}$, detailed histograms showing their distributions are provided. These are interesting because in the existing literature (e.g., 10,11,12 ) these distributions are only considered at several distinct global densities, whereas we show them for all densities. Each of our histograms is constructed by varying the global density $k$ between 0.0 and 1.0, calculating the average speed, the average space gap and the median time gap for each simulation run. A simulation run consists of $5 \times 10^{4} \mathrm{~s}$ (with a transient period of $500 \mathrm{~s}$ ) on systems of 300 cells, varying the density in 150 steps.

All the experiments were carried out with our Java software "Traffic Cellular Automata", which can be found at http://smtca.dyns.cx 13 .

\section{Velocity dependent randomization}

In this section, the rule set of the VDR-TCA model is explained, followed by a an overview of the model's tempospatial behavior and its related macroscopic quantities (i.e., the fundamental diagrams and the distributions of the speeds and the space and time gaps) under normal conditions.

\subsection{The VDR-TCA's rule set}

As indicated before, we focus our research on the VDRTCA model for the implementation of the car-following behavior. The following equations (based on [3] form its rule set; the rules are applied consecutively to all vehicles in parallel (i.e., synchronous updates):

R1: determine stochastic noise

$$
\left\{\begin{array}{l}
v_{i}(t-1)=0 \Longrightarrow p^{\prime} \leftarrow p_{0}, \\
v_{i}(t-1)>0 \Longrightarrow p^{\prime} \leftarrow p,
\end{array}\right.
$$

R2: acceleration and braking

$$
v_{i}(t) \leftarrow \min \left\{v_{i}(t-1)+1, g_{s_{i}}(t-1), v_{\max }\right\},
$$

R3: randomization

$$
\xi_{i}(t)<p^{\prime} \Longrightarrow v_{i}(t) \leftarrow \max \left\{0, v_{i}(t)-1\right\},
$$

R4: vehicle movement

$$
x_{i}(t) \leftarrow x_{i}(t-1)+v_{i}(t)
$$

In the above equations, $v_{i}(t)$ is the speed of vehicle $i$ at time $t$ (i.e., in the current updated configuration of the system), $v_{\max }$ is the maximum allowed speed, $g_{s_{i}}$ denotes the space gap of vehicle $i$ and $x_{i} \in\{1, \ldots, K\}$ an integer number denoting its position in the lattice. In the third rule, equation (10), $\xi_{i}(t) \in[0,1$ [ denotes a uniform random number (specifically drawn for vehicle $i$ at time $t$ ) and $p^{\prime}$ is the stochastic noise parameter, dependent on the vehicle's speed ( $p_{0}$ is called the slow-to-start probability and $p$ the slowdown probability, with $\left.p_{0}, p \in[0,1]\right)$.

In a nutshell, rule $R 1$, equation (8), determines the correct velocity dependent randomization. Rule $R 2$, equation (9), states that a vehicle tries to increase its speed at each time step, as long as it hasn't reached its maximal speed and it has enough space headway. It also states that when a vehicle hasn't enough space headway, it abruptly adapts its speed in order to prevent a collision with the leading vehicle. The randomization parameter determined in equation (8), is now used in rule $R 3$, equation (10), to introduce a stochastic component in the system: a vehicle will randomly slow down with probability $p^{\prime}$. The last rule $R 4$, equation (11), isn't actually a 'real' rule; it just allows the vehicles to advance in the system.

\subsection{Normal behavioral characteristics}

Depending on their speed, vehicles are subject to different randomizations: typical metastable behavior results when $p_{0} \gg p$, meaning that stopped vehicles have to wait longer before they can continue their journey (i.e, they are 'slowto-start'). This has the effect of a reduced outflow from a jam, so that, in a closed system, this leads to an equilibrium and the formation of a compact jam.

A 'capacity drop' takes place at the critical density, where traffic in its vicinity behaves in a metastable manner. This metastability is characterized by the fact that a sufficiently large disturbance of the fragile equilibrium can cause the flow to undergo a sudden decrease, corresponding to a first-order phase transition. The state of very high flow is then destroyed and the system settles into a phase separated state with a large megajam and a free-flow zone 3.14. The large jam will persist as long as the density is not significantly lowered, meaning that recovery of traffic from congestion thus shows a hysteresis phenomenon [15].

If we look at the distribution of the vehicles' speeds, we get the histogram in Fig. 2] Here we can clearly see the distinction between the free-flowing and the congested phase: the space mean speed remains constant at a high value, then encounters a sharp transition (i.e., the capacity drop), resulting in a steady declination as the global density increases. Once the compact jam is formed, the dominating speed quickly becomes zero (because vehicles are standing still inside the jam).

Considering the distribution of the vehicles' space gaps, we get Fig. 3 because of their tight coupling in the VDRTCA's rule set, the courses of both the space mean speed and the average space gaps are similar. Although the space gaps are rather large for low densities, at the critical density $k_{c}$ they leave a small cluster around an optimal value of five cells. This corresponds to the necessary minimal space gap in order to travel at the maximum speed, avoiding a collision with the leader. An important observation is that no recorded space gaps exist between this cluster of five cells and the space gaps of zero cells inside the 

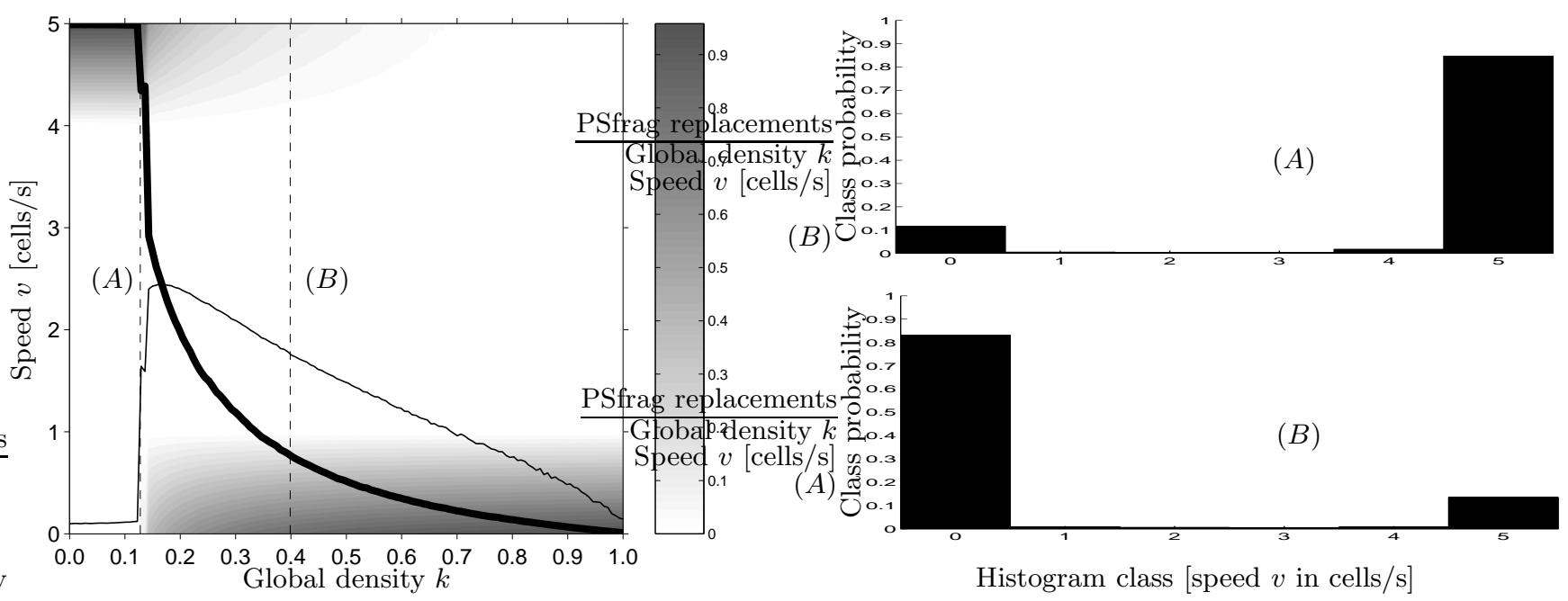

Fig. 2. The distribution of the vehicles' speeds $v$, as a function of the global density $k$ in the VDR-TCA (with $p_{0}=0.5$ and $p=0.01$ ). In the contourplot to the left, the thick solid line denotes the space mean speed, whereas the thin solid line shows its standard deviation. The grey regions denote the probability densities. The histograms $(A)$ and $(B)$ to the right, show two cross sections made in the left contourplot at $k=0.1325$ and $k=0.4000$ respectively: for example, in $(A)$, the high concentration of probability mass at the histogram class $v=5$ cells/s corresponds to the dark region in the upper left corner of the contourplot.

compact jam. This means that there is a distinct phase separation taking place once beyond the critical density: vehicles are either completely in the free-flowing regime, or they are in the compact jam.

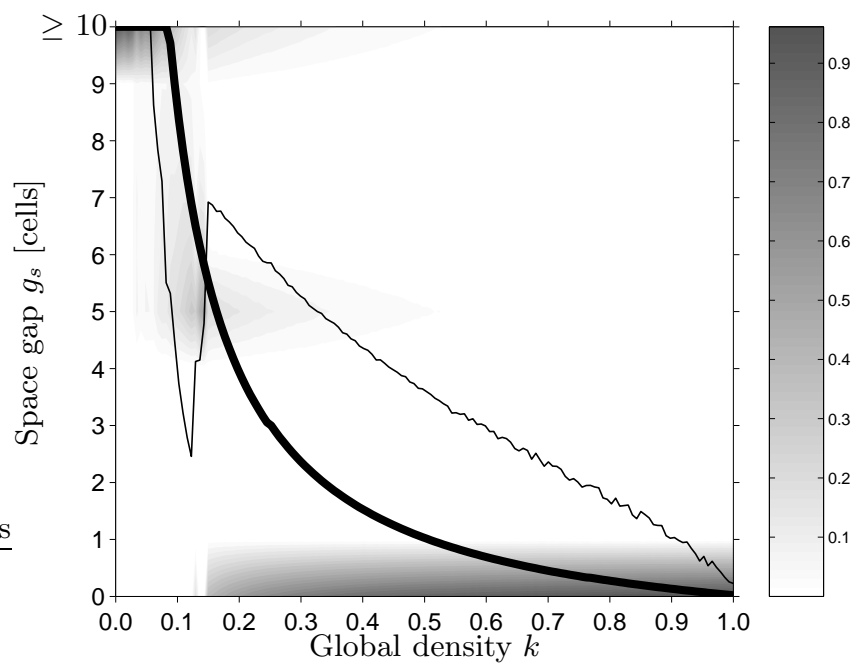

Fig. 3. The distribution of the vehicles' space gaps $g_{s}$, as a function of the global density $k$ in the VDR-TCA (with $p_{0}=$ 0.5 and $p=0.01$ ). The thick solid line denotes the average of all the vehicles' space gaps, whereas the thin solid line shows its standard deviation. The grey regions denote the probability densities.

Figure 4 shows the distribution of the vehicles' time gaps: when traffic is in the free-flowing regime, time gaps are high, but finite. As the critical density is approached, the median time gap first decreases (because the vehicles' speeds remain the same but their space gaps decrease). Once beyond the critical density, it increases towards infinity because vehicles come to a full stop inside the compact jam. Just as with the space gaps, we can also observe a small cluster around an optimal value. This optimal time gap, is the time needed to travel the distance formed by the optimal space gap, at the maximum speed. This means that $\bar{g}_{t}=\bar{g}_{s} \div v_{\max }=1 \mathrm{~s}$. Because the VDRTCA incorporates stochastic noise, the median optimal time gap lies somewhat above the previously calculated value $\left(\bar{g}_{t} \approx 1.2 \mathrm{~s}\right)$.

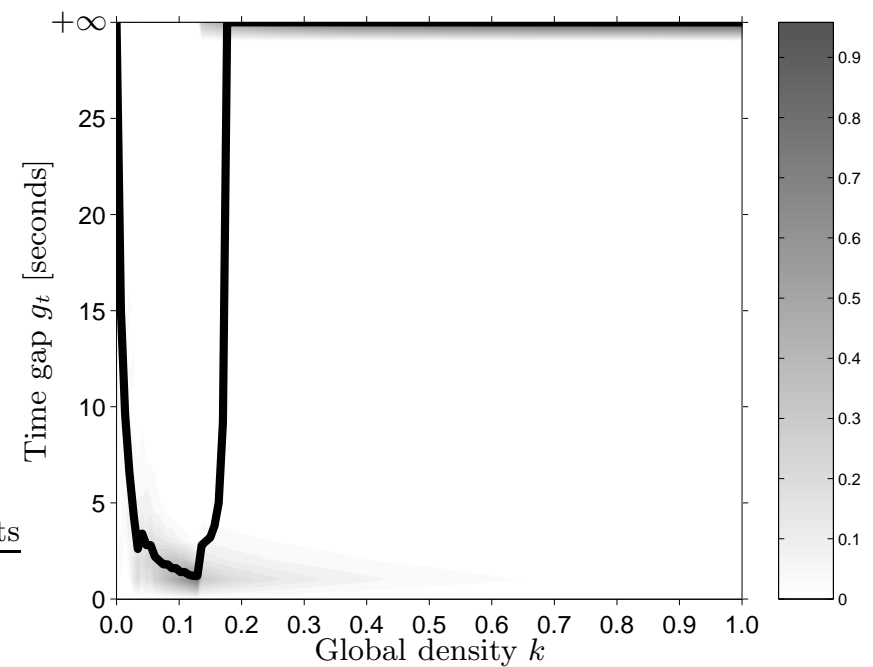

Fig. 4. The distribution of the vehicles' time gaps $g_{t}$, as a function of the global density $k$ in the VDR-TCA (with $p_{0}=$ 0.5 and $p=0.01$ ). The thick solid line denotes the median of all the vehicles' time gaps. The grey regions denote the probability densities. 


\section{More complex system dynamics}

Most of the previous research dealt with the study of the behavioral characteristics of the VDR-TCA model operating under normal conditions. We now turn our attention to the specific case in which the model's parameters take on extreme values $p_{0} \ll p$, more specifically considering the limiting case where $p_{0}=0.0$ and $p=1.0$.

We will first look at the change in tempo-spatial behavior when $p$ is increased towards 1.0, at which point a peculiar behavior is established in the system. We study the qualitative effects that the VDR-TCA's rule set has on individual vehicles, and discuss shortly the prevailing initial conditions. This is followed by a quantitative analysis using the $(k, q)$ fundamental diagram, leading us to the discovery of four distinct phases, having a non-concave region with forward propagating density waves. More elaborate explanations are given based on the histograms of the space mean speed, the average space gap, and the median time gap. The section concludes with an analysis of the observations of the tempo-spatial behavior of the system in each of the four different phases (i.e., traffic regimes).

\subsection{Increasing the stochastic noise $p$}

Let us first consider the case in which $p_{0}=0.0$ and where we vary $p$ between 0.0 and 1.0. Figure 5 shows a timespace diagram where we simulated a system consisting of 300 cells. As time advances (over a period of $580 \mathrm{~s}$ ), the slowdown probability $p$ is steadily increased from 0.0 to 1.0 (the global density $k$ was set to 0.1667 which is slightly below the critical density for a system with stochastic noise).

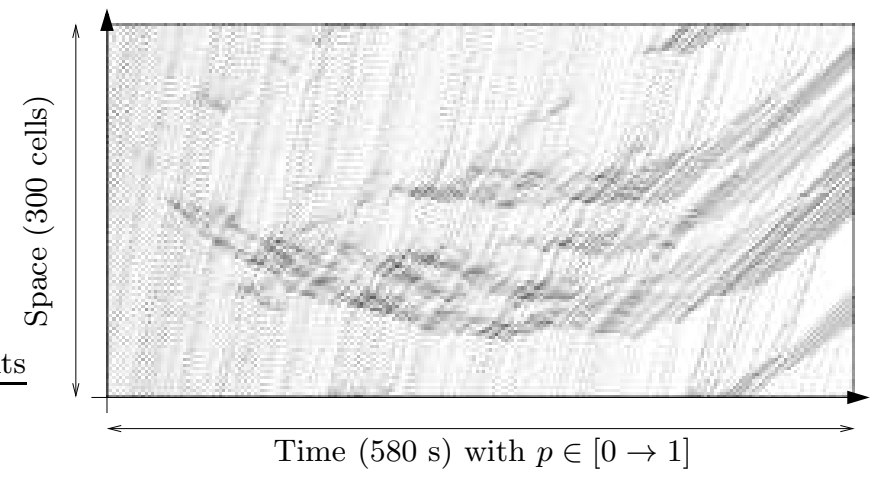

Fig. 5. A time-space diagram showing a system with a lattice of 300 cells (corresponding to $2.25 \mathrm{~km}$ ); the visible time horizon is 580 seconds. As time advances, the slowdown probability $p$ varies between 0.0 and 1.0 (the slow-to-start probability $p_{0}$ was fixed at 0.0 ). The system's global density $k$ is 0.1667 .

When $p$ is very low, vehicles can keep driving in the free-flowing regime. As $p$ is increased, small unstable jams occur. Increasing $p$ even further, leads to even more pronounced jams. An important observation is that the propagation speed of these backward moving congestion waves increases; when $p$ reaches approximately 0.5 , their speed equals 0 , meaning that jams stay fixed at a position. Note that as these jams are unstable, they can be created or dissolved at arbitrary locations. Finally, as $p$ tends to 1.0, we can see the emergence of forward propagating congestion waves; 'density' is now being carried in the direction of the traffic flow. These forward propagating waves form 'moving blockades' that trap vehicles, which in turn leads to tightly packed clusters of vehicles that move steadily (but at a much slower pace than in the free-flowing regime). Note that this 'clustering' behavior is different from platooning, which typically occurs when vehicles are driving close to each other at relatively high speeds [16. In our case, the clusters of vehicles advance more slowly.

\subsection{Qualitative effects of the rule set}

From now on, we only consider the limiting case where $p_{0}=0.0$ and $p=1.0$. Let us now study the influence of the VDR-TCA's rule set on an individual vehicle $i$. Assuming $v_{i} \in\left\{0, \ldots, v_{\max }\right\}$, the rules described in section 3.1 lead to the following four general cases:

- Case (1) with $v_{i}(t-1)=0$ and $g_{s_{i}}(t-1)=0$

In this case, the vehicle is residing inside a jam and rule $R 2$ (acceleration and braking) plays a dominant part: the vehicle's speed $v_{i}(t)$ remains 0 .

- Case (2) with $v_{i}(t-1)=0$ and $g_{s_{i}}(t-1)>0$

This situation may arise when, for example, a vehicle is at a jam's front. According to rule $R 1$, the stochastic noise parameter $p^{\prime}$ becomes 0.0 . Rule $R 2$ then results in an updated speed $v_{i}(t)=\min \left\{1, g_{s_{i}}(t-1)\right\}=1$. Because there is no stochastic noise present in this case, rule $R 3$ does not apply and the vehicle always advances one cell.

- Case (3) with $v_{i}(t-1)>0$ and $g_{s_{i}}(t-1)=0$

Because there is no space in front of the vehicle, it has to brake in order to avoid a collision. Rule $R 2$ consequently abruptly decreases the vehicle's speed $v_{i}(t)$ to 0 , as the vehicle needs to stop.

- Case (4) with $v_{i}(t-1)>0$ and $g_{s_{i}}(t-1)>0$

This case deserves special attention, as there are now two discriminating possibilities:

- Case (4a) with $v_{i}(t-1)<g_{s_{i}}(t-1)$

According to rule $R 1$, the stochastic noise $p^{\prime}$ becomes 1.0. Because the vehicle's speed is strictly less than its space gap, rule $R 2$ becomes $v_{i}(t) \leftarrow$ $\min \left\{v_{i}(t-1)+1, v_{\max }\right\}$. Finally, rule $R 3$ is applied which always reduces the speed calculated in rule $R 2$ (constrained to 0 ). In order to understand what is happening, consider the speeds $v_{i}(t-1)$ and $v_{i}(t)$ in the following table: 


\begin{tabular}{lll}
$v_{i}(t-1)$ & & $v_{i}(t)$ \\
\hline$v_{\max }$ & $\longrightarrow$ & $v_{\max }-1$ \\
$v_{\max }-1$ & $\longrightarrow$ & $v_{\max }-1$ \\
$v_{\max }-2$ & $\longrightarrow$ & $v_{\max }-2$ \\
$\vdots$ & & $\vdots$ \\
2 & $\longrightarrow$ & 2 \\
1 & $\longrightarrow$ & 1
\end{tabular}

We can clearly see that the maximum speed a vehicle can travel at, is constrained by $v_{\max }-1$, which corresponds to $v_{\max }-p^{\prime}$ [17. From the table it follows that all vehicles traveling at $v_{i}<v_{\max }$ can neither accelerate nor decelerate: the vehicles' current speed is kept.

- Case (4b) with $v_{i}(t-1) \geq g_{s_{i}}(t-1)$

Just as in the previous case (4a), the stochastic noise $p^{\prime}$ becomes 1.0. Rule $R 2$ now changes to $v_{i}(t) \leftarrow$ $g_{s_{i}}(t-1)$. Because rule $R 3$ is always applied, this results in $v_{i}(t)$ actually becoming $g_{s_{i}}(t-1)-1$ instead of just $g_{s_{i}}(t-1)$. So a vehicle always slows down too much (as opposed to solely avoiding a collision with its leader).

Conclusion: considering the previously discussed four general cases, the most striking feature is that, according to case (4a), in a VDR-TCA model with $p_{0}=0.0$ and $p=1.0$, a moving vehicle can never increase its speed, i.e., $v_{i}(t) \leq v_{i}(t-1)$.

\subsection{Effects of the initial conditions}

As already mentioned, in this paper we study the limiting case where $p_{0}=0.0$ and $p=1.0$. This case is special, in the sense that the system's behavior for light densities is extremely dependent on the initial conditions.

After the system has settled into an equilibrium, the resulting flow is limited by the fact that the maximum speed of a vehicle in the system is always equal to $v_{\max }-1$. As proven in section 4.2 vehicles can never accelerate, which means that the slowest car in the system determines the maximum possible flow. Therefore, if the system (with $v_{\max }=5$ cells $/ \mathrm{s}$ ) is initialized with a homogeneous distribution of the vehicles, then all of them will travel at $v_{\max }-1=4$ cells $/ \mathrm{s}$. If the system is initialized randomly, all vehicles will now travel at $v_{\max }^{\prime}-1$ cells/s with $v_{\max }^{\prime}$ the speed of the slowest vehicle in the system.

\subsection{Quantitative analysis}

Whereas the previous sections dealt with the effects of the VDR-TCA's changed rule set and the role of the initial conditions, this section considers the effects on the $(k, q)$ fundamental diagram (i.e., three phase transitions and a non-concave region), as well as on the histograms of the space mean speed, the average space gap and the median time gap.

\subsubsection{Effects on the fundamental diagrams}

Most existing $(k, q)$ fundamental diagrams related to traffic flow models, show a concave course (although some (pedagogic) counter-examples such as 4, 5, 6,7,8, 9] exist). Non-concavity (i.e., convexity) of a function $f$ is defined as $\forall x_{1}, x_{2} \in \operatorname{dom} f \mid f\left(\frac{1}{2}\left(x_{1}+x_{2}\right)\right) \leq \frac{1}{2} f\left(\left(x_{1}\right)+f\left(x_{2}\right)\right)$. The property of concavity also holds true for the VDRTCA model operating under normal conditions. However, when $p_{0} \ll p$, this is no longer the case: in the limit where $p_{0}=0.0$ and $p=1.0$, the fundamental diagram exhibits two distinct sharp peaks. Between these peaks, there exists a region $(I I)+(I I I)$ where the fundamental diagram has a convex shape, as can be seen from Fig. [6] In region (III), traffic flow has a tendency to improve with increasing density. Note that we ignore the hysteretic behavior of the fundamental diagrams, as it gives no relevant contribution to the results of our experiments.

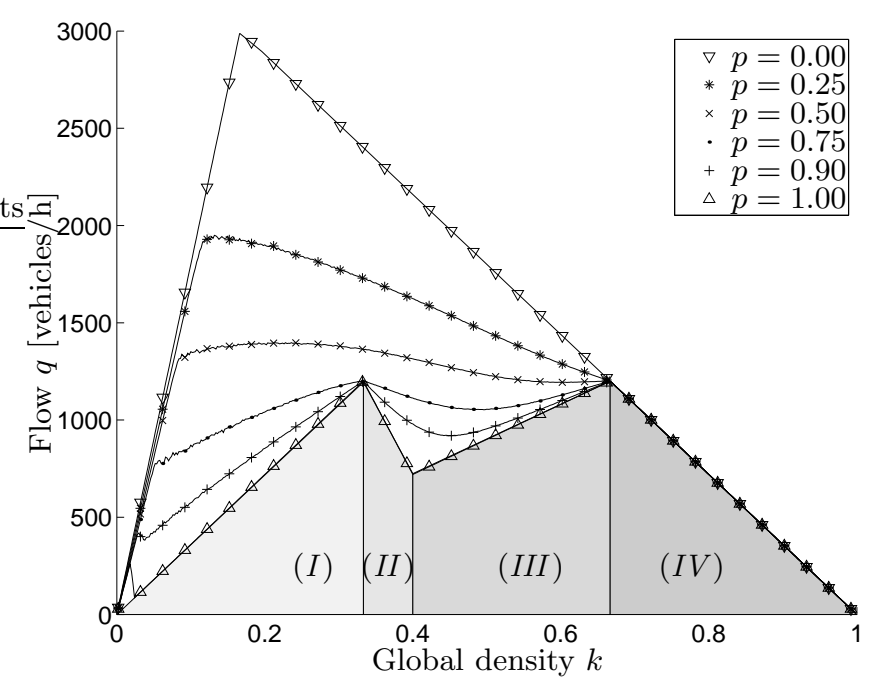

Fig. 6. The $(k, q)$ fundamental diagrams of the VDR-TCA with a fixed slow-to-start probability $p_{0}=0.0$ (the slowdown probability $p$ is increased from 0.0 to 1.0$)$. In the limiting case where $p=1.0$, four distinct density regions $(I)-(I V)$ appear.

As the slowdown probability $p$ is increased from 0.0 to 1.0, the critical density - at which the transition from the free-flowing regime occurs - is shifted to lower values (note that the magnitude of the capacity drop also diminishes). For a global density $k=\frac{1}{6}$, we can see that the speed of the backward propagating congestion waves increases, just as was visible in the time-space diagram of Fig. [5 The speed of these characteristics is defined as $\partial q / \partial k$ (i.e., the tangent to the fundamental diagram), and when $p \approx 0.5$, the sign of the speed is reversed, leading to the earlier mentioned forward propagating density waves. Furthermore, as is apparent in Fig. [6] for high densities there exists a region $(I V)$ in which the flow $q$ is only dependent on $k$ and not on the stochastic noise $p$ : all the measurements coincide on this heavily congested branch. 
Increasing the slowdown probability $p$ has also an effect on the space mean speed in the free-flowing regime. As already stated in section 3.2 this speed is equal to $v_{\max }-$ $p$ [17. The average maximum speed in the free-flowing regime shifts downwards, reaching a value of 4 cells/s when $p=1.0$. From then on, there are two regions $(I)$ and $(I I I)$ where the average speed remains constant. Note that, to be precise, region $(I)$ actually contains a small capacity drop at a very low density, but we ignore this effect, thus treating region $(I)$ in an overall manner.

\subsubsection{Effects on the histograms}

The distribution of the vehicles' speeds is shown in Fig. 7 where we can clearly observe two 'probability plateaus'. In the first region $(I)$, vehicles' speeds are highly concentrated in a small region around $\bar{v}_{s}=1 \mathrm{cell} / \mathrm{s}$. As the global density increases in region $(I I)$, the space mean speed declines until it reaches region (III) where the second plateau is met at $\bar{v}_{s}=0.5 \mathrm{cell} / \mathrm{s}$. From then on, it steadily decreases, reaching zero at the jam density $\left(k_{j}=1.0\right)$. Note that in region $(I)$, the standard deviation is zero, whereas it is non-zero but constant in region (III). This means that vehicles in the former region all drive at the same speed; in the latter region they drive at speeds alternating between 0 and 1 cell/s (i.e., stop-and-go traffic).

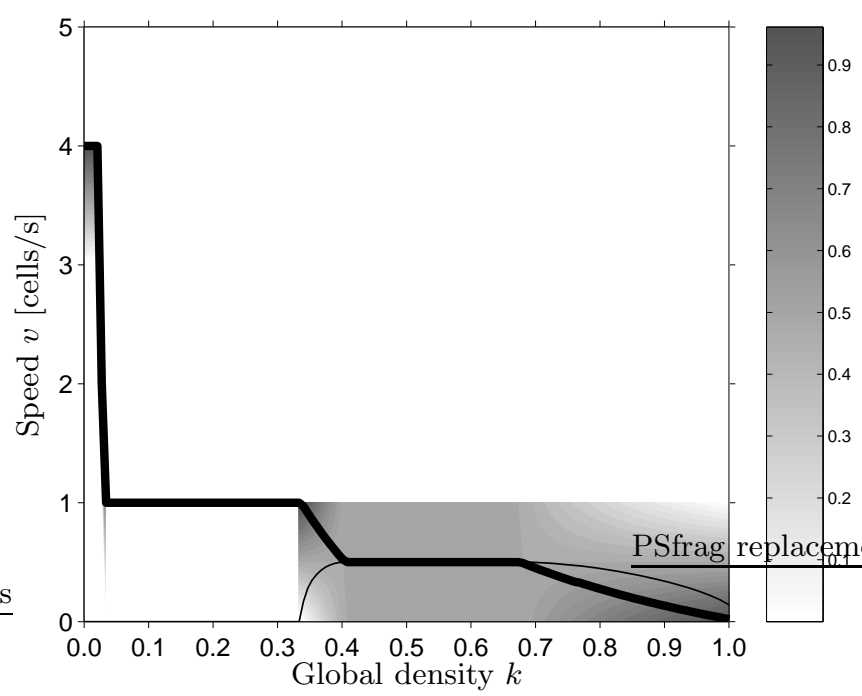

Fig. 7. The distribution of the vehicles' speeds $v$, as a function of the global density $k$ in the VDR-TCA (with $p_{0}=0.0$ and $p=1.0$ ). The thick solid line denotes the space mean speed, whereas the thin solid line shows its standard deviation. The grey regions denote the probability densities.

Considering the steep descending curve at the beginning of region $(I)$, we state that although vehicles can drive at $v=v_{\max }-1=4$ cell/s under free-flowing conditions, they nonetheless all slow down as soon as at least one vehicle has a too small space gap. In other words, when $g_{s_{i}} \leq v_{\max }$, case (4b) from section 4.2 applies and the vehicle slows down. This leads to a chain reaction of vehicles slowing down, because vehicles can never accelerate.

Under normal operating conditions (i.e., $p_{0} \gg p$ ), a vehicle's average speed and average space gap show a high correlation in the congested density region beyond the critical density. This can be seen from the similarity between the histogram curves in Figs. 22 and 3. In high contrast with this, is the distribution of the vehicles' space gaps as in Fig. 8, which shows a different scenario. More or less similar to the vehicles' speeds, we can observe the formation of three plateaus of constant space gaps in certain density regions. These plateaus are located at 2, 1 and 0 cells for density regions $(I),(I I I)$, and $(I V)$ respectively. Note that the average space gaps themselves are not constant in these regions, as opposed to the space mean speed. This is due to the fact that vehicles encounter waves of stop-and-go traffic, whereby the frequency of these waves increases as the global density is augmented.

Another observation that we can make, is that the standard deviation goes to zero at the transition point between density regions $(I)$ and $(I I)$. This means that, as expected, the traffic flow at this point consists of completely homogeneous traffic, in which all the vehicles drive with the same space gap $g_{s}=2$ cells (and as already mentioned, with the same speed $v=1 \mathrm{cell} / \mathrm{s})$.

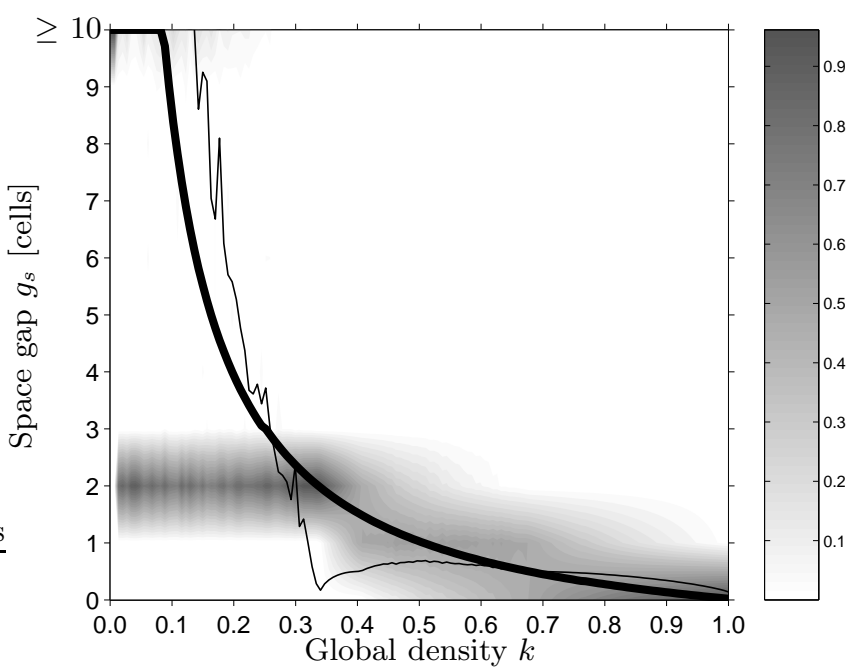

Fig. 8. The distribution of the vehicles' space gaps $g_{s}$, as a function of the global density $k$ in the VDR-TCA (with $p_{0}=$ 0.0 and $p=1.0$ ). The thick solid line denotes the average of all the vehicles' space gaps, whereas the thin solid line shows its standard deviation. The grey regions denote the probability densities.

Just as with the previous histograms, there also appear to be plateaus of concentrated probability mass in the distribution of the vehicles' time gaps in Fig. 9 As

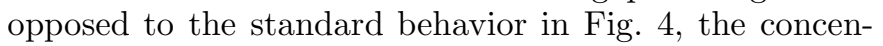
tration in the first region $(I)$ is more elongated and more or less completely flat. This is expected because the ma- 
jority of the space mean speeds and the average space gaps remain constant in this region. Furthermore, once the first phase transition occurs, the median time gap $\bar{g}_{t} \rightarrow+\infty$ as the space mean speeds and average space gaps tend to zero. This is expressed as the existence of a non-neglibible cluster of probability mass at the top of the histogram in Fig. 9 the concentration is formed by vehicles that are encountering the earlier mentioned stop-and-go waves, resulting in the fact that their time gaps periodically tend towards infinity.

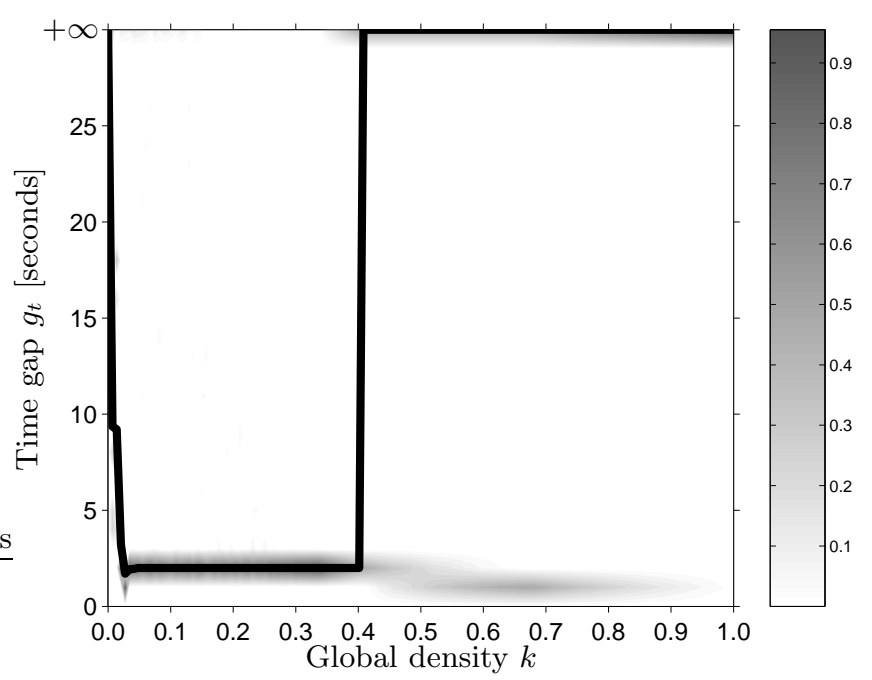

Fig. 9. The distribution of the vehicles' time gaps $g_{t}$, as a function of the global density $k$ in the VDR-TCA (with $p_{0}=$ 0.0 and $p=1.0$ ). The thick solid line denotes the median of all the vehicles' time gaps. The grey regions denote the probability densities.

\subsection{Typical tempo-spatial behavior}

Studying the $(k, q)$ fundamental diagrams (Fig. 6) in the previous section, we saw the emergence of four distinct density regions $(I)-(I V)$ as $p \rightarrow 1.0$ (formed by the $\triangle$ symbols). In this section, we discuss the tempo-spatial properties that are intrinsic to these regions, relating them to the previously discussed histograms.

As the global density $k$ is increased, the system undergoes three consecutive phase transitions (between these four traffic regimes). The next four paragraphs detail the effects that appear in each density region (i.e., traffic regime), as well as the transitions that occur between them.

\subsubsection{Region $(I)$ - free-flowing traffic [FFT]}

According to the histogram in Fig. 7 we can see that the speed of all the vehicles is the same (namely 1 cell/s) for moderately low densities. Although the standard deviation of the space mean speed is zero, this is not the case

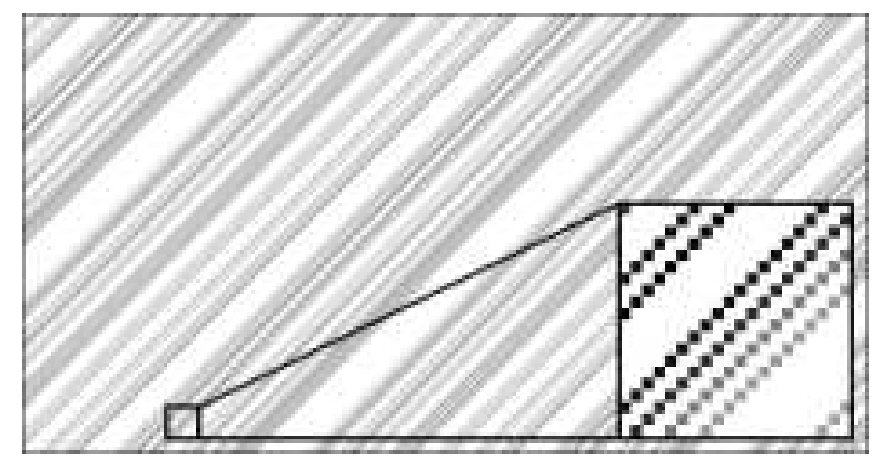

Fig. 10. Time-space diagram of the VDR-TCA model with $p_{0}=0.0$ and $p=1.0$. The shown lattice contains 300 cells (corresponding to $2.25 \mathrm{~km}$ ), the visible time horizon is 580 seconds. The density $k=0.2$, corresponding to observations in density region $(I)$, labeled free-flowing traffic (FFT).

for the average space gap, explaining its rather 'nervous' behavior in Fig. 8

As the global density increases, the transition point between regions $(I)$ and $(I I)$ is reached. At this point, each vehicle $i$ has a speed $v_{i}=1 \mathrm{cell} / \mathrm{s}$, and a space gap $g_{s_{i}}=2$ cells. Because $v_{i}<g_{s_{i}}$, case (4a) from section 4.2 applies. Using equations (1) and (6), we can calculate the corresponding density:

$$
k_{(I) \rightarrow(I I)}=\bar{h}_{s}^{-1}=\left(\bar{L}+\bar{g}_{s}\right)^{-1}=\frac{1}{3} .
$$

Although the maximum speed any vehicle can (and will) travel at is limited to 1 cell/s, we still call this state 'free-flowing traffic' (FFT) because no congestion waves are present in the system and none of the vehicles has to stop (see the time-space diagram in Fig. 10).

\subsubsection{Region (II) - dilutely congested traffic [DCT]}

If we increase the density to $k=\frac{1}{3}+\frac{1}{K}$ (i.e., adding one vehicle at the transition point), a new traffic regime is entered. In this regime, each extra vehicle leads to a backward propagating mini-jam of 3 stop-and-go cycles (see Fig. (11), bringing us to the description of 'dilutely congested traffic' (DCT).

In order to calculate the second transition point, we again observe the histogram of Fig. 8 A vehicle's space gap now alternates between 1 and 2 cells in density region $(I I)$. And because its speed alternates between 0 and $1 \mathrm{cell} / \mathrm{s}$ respectively, the vehicles' motions are controlled by cases (2) and (4a) from section 4.2 This means that stopped vehicles accelerate again in the next time step, after which they have to stop again, and so indefinitely repeating this cycle of stop-and-go behavior.

Consider now a pair of adjacent driving vehicles $i$ and $j$; it then follows from equation (11) that $h_{s_{i}}=1+1=$ 2 cells and $h_{s_{j}}=1+2=3$ cells. So each pair of vehicles 'occupies' 5 cells in the lattice, or 2.5 cells on average per vehicle. This leads to the second transition point being located at: 


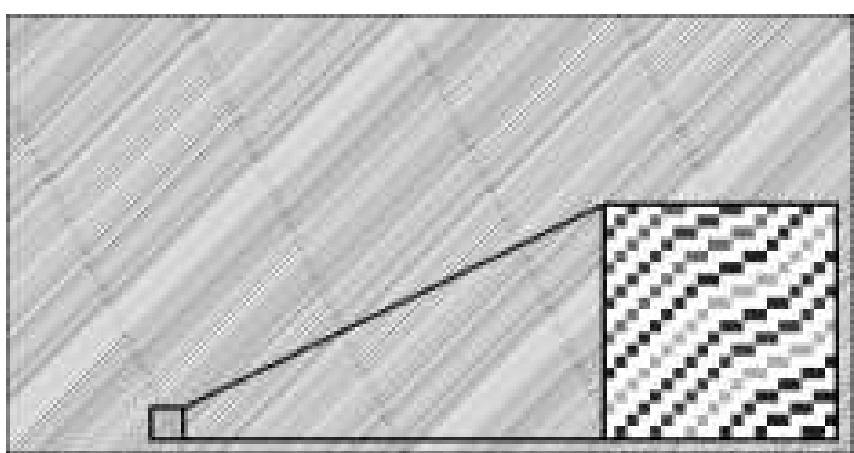

Fig. 11. Time-space diagram of the VDR-TCA model with $p_{0}=0.0$ and $p=1.0$. The shown lattice contains 300 cells (corresponding to $2.25 \mathrm{~km}$ ), the visible time horizon is 580 seconds. The density $k=\frac{1}{3}+\frac{1}{K}$, corresponding to observations in density region (II), labeled dilutely congested traffic (DCT).

$$
k_{(I I) \rightarrow(I I I)}=\bar{h}_{s}^{-1}=\frac{1}{2.5}=0.4 .
$$

As the density is increased towards $k_{(I I) \rightarrow(I I I)}$, the space mean speed decreases non-linearly. At the transition point itself, $\bar{v}_{s}=0.5$ cells $/ \mathrm{s}$ and the system is now completely dominated by backward propagating dilute jams.

\subsubsection{Region (III) - densely advancing traffic [DAT]}

Adding one more vehicle at the transition point between regions $(I I)$ and $(I I I)$, leads to surprising behavior: a forward moving jam emerges, traveling at a speed of 0.5 cells $/ \mathrm{s}$ (see Fig. 12). Another artefact is that the cycle of alternating space gaps of 1 and 2 cells is broken, with the introduction of a zero space gap at the location of this new 'jam'. When the density reaches the third transition point, the system is completely filled with these forward moving 'jams' of dense traffic, leading to the description of 'densely advancing traffic' (DAT). Because the available space is more optimally used by the vehicles, an increase of the density thus has a (temporary) beneficial effect on the global flow measured in the system. This kind of behavior of forward moving density structures can also be observed in some models of anticipatory driving [16].

At the transition point itself, all vehicles exhibit the same behavior, comparable to the behavior at the second transition point. Traffic is more dense, as can be seen in the distribution of the space gaps in Fig. 8 all vehicles have alternatingly $g_{s}=0$ and $g_{s}=1$ cell (with corresponding speeds of 0 and 1 cell/s respectively). One pair of adjacent driving vehicles $i$ and $j$ thus 'occupies' $h_{s_{i}}+h_{s_{j}}=(1+0)+(1+1)=3$ cells, or 1.5 cells on average per vehicle. The third transition point thus corresponds to:

$$
k_{(I I I) \rightarrow(I V)}=\bar{h}_{s}^{-1}=\frac{1}{1.5}=\frac{2}{3} .
$$

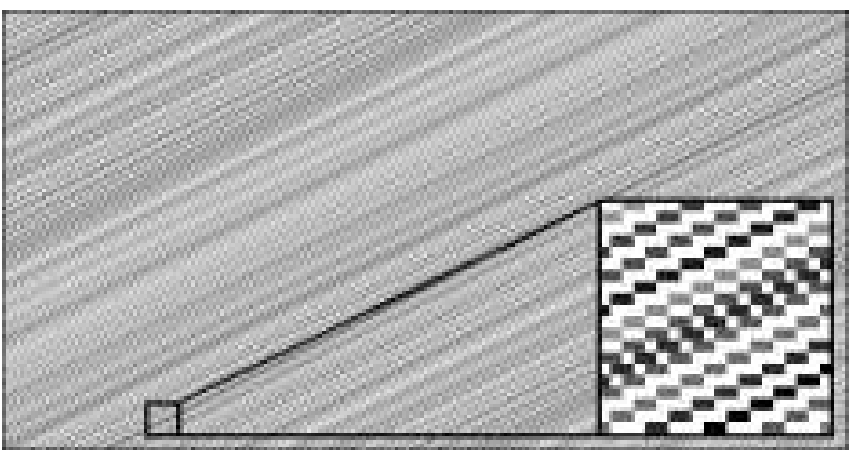

Fig. 12. Time-space diagram of the VDR-TCA model with $p_{0}=0.0$ and $p=1.0$. The shown lattice contains 300 cells (corresponding to $2.25 \mathrm{~km}$ ), the visible time horizon is 580 seconds. The density $k=0.4+\frac{1}{K}$, corresponding to observations in density region (III), labeled densely advancing traffic (DAT).

\subsubsection{Region (IV) - heavily congested traffic [HCT]}

Finally, as the system's global density is pushed towards the jam density, each extra vehicle introduces at any point in time a backward propagating jam, consisting of a block of five consecutively stopped vehicles (see Fig. 13). The pattern of stable stop-and-go traffic gets destroyed, as vehicles remain stopped inside jams for longer time periods (cfr. density region $(I V)$ in the distribution of the time gaps in Fig. 9), leading to the description of this traffic regime as 'heavily congested traffic' (HCT).

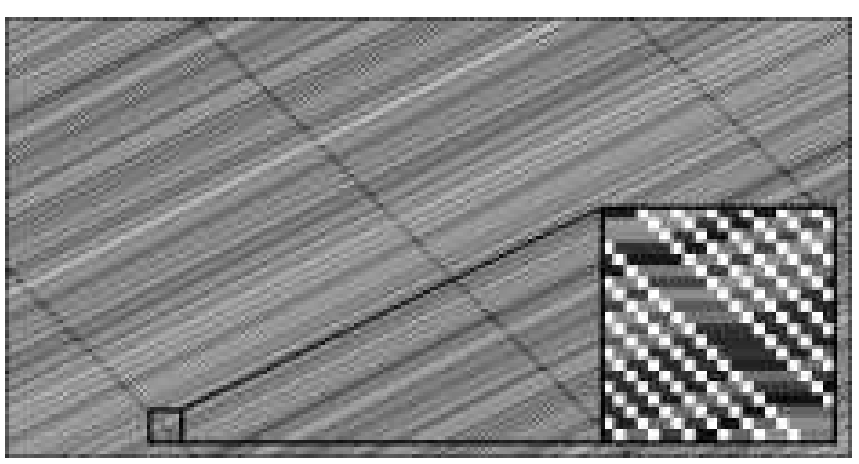

Fig. 13. Time-space diagrams of the VDR-TCA model with $p_{0}=0.0$ and $p=1.0$. The shown lattice contains 300 cells (corresponding to $2.25 \mathrm{~km}$ ), the visible time horizon is 580 seconds. The density $k=\frac{2}{3}+\frac{1}{K}$, corresponding to observations in density region $(I V)$, labeled heavily congested traffic (HCT).

\section{Comparison with existing literature}

A final word should be said about the behavior of the four phases discussed in this paper. Although the study is based on a well-known traffic flow model (i.e., the VDRTCA model), it seems that this behavior is drastically different from that observed in real-life traffic. The research 
elucidated in this paper, is therefore relevant as it can be compared to phase transitions in other types of granular media, described by cellular automata.

Relating our findings to similar considerations in literature, we note the following aspects: Grabolus [5] gives a thorough discussion of several variants of the STCA, including so-called 'fast-to-start' TCA models that give rise to forward propagating density waves; the study refers to the two-fluid theory [18 as a possible explanation of this phenomenon. Gray and Griffeath [6] discuss the origin of non-concavity in the $(k, q)$ fundamental diagram, where they propose an explanation in which the rear end of a jam is unstable, whereas its front is stable and growing. Leveque [7] incorporates a non-concave fundamental diagram itself in a macroscopic model, thereby resembling night driving. Nishinari et al [8] consider the tempo-spatial organization of ants using an ant trail model based on pheromones. In their model, the average speed of the ants varies non-monotonically with their density, leading to an inflection point in the $(k, q)$ fundamental diagram. Zhang [9] questions the property of anisotropy in multi-lane traffic, leading to strikingly similar non-concave $(k, q)$ fundamental diagrams.

Finally, Awazu [4] investigated the flow of various complex particles using a simple meta-model based on Wolfram's CA-rule 184, but extended with rules that govern the change in speed of individual particles. As a result, he classified three types of fundamental diagrams, namely two phases $(2 \mathrm{P})$, three phases $(3 \mathrm{P})$, and four phases $(4 \mathrm{P})$ type systems. Of these types, the 4P-type complex granular particle systems closely resemble the regimes discovered in this paper. Awazu discusses the types of regimes and the transitions between them, using the terminology of 'dilute slugs' for the slow moving jams in the DCTphase (see section 4.5.2), which he calls the 'dilute jamflow state'. Analogously, there are 'advancing slugs' in the 'advancing jam-flow state' (related to our DAT-phase in section 4.5.31) and the fourth phase is called the 'hard jamflow state' (related to our HCT-phase in section 4.5.4).

As stated before, the behavior here is different from that of real-life traffic flows. Awazu expects that these transitions appear if there are more complex interactions between the particles. In the 4P-type systems, attractive forces between close neighbouring particles and an effective resistance on moving neighbouring particles, lead to the realization of the previously discussed flow phases.

\section{Summary}

In this paper, we first showed the behavioral characteristics resulting from the velocity dependent randomization traffic cellular automaton (VDR-TCA) model, operating under normal conditions (i.e., $p_{0} \gg p$ ). Then we investigated the more complex system dynamics that arise from this traffic flow model in the exceptional cases when $p_{0} \ll p$. This behavior was quantitavely compared against the VDR-TCA's normal operation, using classical fundamental diagrams and histograms that show the distribution of the speeds, space, and time gaps.

Our main investigations were primarily directed at the limiting case where $p_{0}=0.0$ and $p=1.0$. We discovered the emergence of four different traffic regimes. These regimes were individually studied using diagrams that show the evolution of their tempo-spatial behavioral characteristics. This resulted in the following classification: freeflowing traffic (FFT), dilutely congested traffic (DCT), densely advancing traffic (DAT), and heavily congested traffic (HCT). Our main conclusions here are:

- all four phases share the common property that moving vehicles can never increase their speed once the system has settled into an equilibrium,

- the DAT regime experiences forward propagating density waves, corresponding to a non-concave region in the system's flow-density relation.

Comparing our results with those in existing literature, we conclude that the work of Awazu [4, dealing with a cellular automaton model of various complex particles, gives the closest resemblance to our four phases.

Note that a more detailed account of our research (including $\left(k, \bar{v}_{s}\right)$ fundamental diagrams, more on the influence of the maximum speed and the use of a suitable order parameter to track the phase transitions) can be found in our technical report [19.

\section{Acknowledgements}

S. Maerivoet would like to thank dr. Andreas Schadschneider for his insightful comments and feedback when writing this paper.

Our research is supported by: Research Council KUL: GOAMefisto 666, GOA-AMBioRICS, several PhD/postdoc \& fellow grants,

FWO: PhD/postdoc grants, projects, G.0240.99 (multilinear algebra), G.0407.02 (support vector machines), G.0197.02 (power islands), G.0141.03 (identification and cryptography), G.0491.03 (control for intensive care glycemia), G.0120.03 (QIT), G.0452.04 (new quantum algorithms), G.0499.04 (robust SVM), research communities (ICCoS, ANMMM, MLDM),

AWI: Bil. Int. Collaboration Hungary/Poland,

IWT: PhD Grants, GBOU (McKnow),

Belgian Federal Science Policy Office: IUAP P5/22 ('Dynamical Systems and Control: Computation, Identification and Modelling', 2002-2006), PODO-II (CP/40: TMS and Sustainability),

EU: FP5-Quprodis, ERNSI, Eureka 2063-IMPACT, Eureka 2419-FliTE,

Contract Research/agreements: ISMC/IPCOS, Data4s, TML, Elia, LMS, Mastercard. 


\section{References}

1. Kai Nagel and Michael Schreckenberg. A cellular automaton model for freeway traffic. Journal de Physique I France, 2:2221-2229, 1992.

2. Kai Nagel. Life-times of simulated traffic jams. International Journal of Modern Physics C, 5(3):567-580, 1994.

3. R. Barlović, L. Santen, A. Schadschneider, and M. Schreckenberg. Metastable states in cellular automata for traffic flow. European Physics Journal, B5(793), 1998.

4. Akinori Awazu. Cellular automaton rule $184++$ C. A simple model for the complex dynamics of various particles flow. Physics Letters A, 261:309-315, October 1999.

5. Sascha Grabolus. Numerische Untersuchungen zum NagelSchreckenberg-Verkehrsmodell und dessen Varianten. Master's thesis, Institut für Theoretische Physik, Universität zu Köln, 2001.

6. Lawrence Gray and David Griffeath. The ergodic theory of traffic jams. Journal of Statistical Physics, 105(3-4):413452, November 2001.

7. Randall J. Leveque. Some traffic flow models illustrating interesting hyperbolic behavior. In Minisymposium on traffic flow,. SIAM Annual Meeting, July 2001.

8. Katsuhiro Nishinari, Debashish Chowdhury, and Andreas Schadschneider. Cluster formation and anomalous fundamental diagram in an ant trail model. Physical Review E, 67:036120-1-036120-11, March 2003.

9. H.M. Zhang. Anisotropic property revisited - does it hold in multi-lane traffic ? Transportation Research B, 37B(6):561-577, July 2003.

10. Debashish Chowdhury, Abhay Pasupathy, and Shishir Sinha. Distributions of time- and distance-headways in the nagel-schreckenberg model of vehicular traffic: Effects of hindrances. European Physics Journal B - Condensed Matter, 5(3):781-786, October 1998.

11. Andreas Schadschneider. Statistical physics of traffic flow. Physica A, (285):101, 2000.

12. Dirk Helbing. Traffic and related self-driven many-particle systems. Review of Modern Physics, 73:1067-1141, October 2001 .

13. Sven Maerivoet. Traffic Cellular Automata. Java software tested with JDK 1.3.1, 2004.

(URL : http://smtca.dyns.cx).

14. Debashish Chowdhury, Ludger Santen, and Andreas Schadschneider. Statistical physics of vehicular traffic and some related systems. Physics Reports, 329:199-329, 2000.

15. R. Barlović, J. Esser, K. Froese, W. Knospe, L. Neubat, M. Schreckenberg, and J. Wahle. Online traffic simulation with cellular automata. Traffic and Mobility : SimulationEconomics-Environment, pages 117-134, July 1999. Institut für Kraftfahrwesen, RWTH Aachen, Duisburg.

16. M.E. Lárraga, J.A. del Río, and A. Schadschneider. New kind of phase separation in a CA traffic model with anticipation. Journal of Physics A: Mathematical and General, (37):3769-3781, 2004.

17. M. Schreckenberg, R.Barlović, W. Knospe, and H. Klüpfel. Statistical physics of cellular automata models for traffic flow. In K. H. Hoffmann and M. Schreiber, editors, Computational Statistical Physics, pages 113-126, Berlin, 2001. Springer.

18. James C. Williams. Two-fluid theory. In Nathan Gartner, Hani Mahmassani, Carroll J. Messer, Henry Lieu, Richard Cunard, and Ajay K. Rathi, editors, Traffic Flow Theory:
A State-of-the-Art Report, pages 6.17-6.29. Federal Highway Administration, Transportation Reseach Board, 1997.

19. Sven Maerivoet and Bart De Moor. Advancing density waves and phase transitions in a velocity dependent randomization traffic cellular automaton. Technical Report 03-111, Katholieke Universiteit Leuven, October 2004. 